\title{
Is linguistics a natural science?
}

\author{
Jan Koster \\ University of Groningen \\ j.koster@rug.nl
}

\section{Language: internal or external?}

Although language uncontroversially has internal, psychological dimensions and external, cultural aspects, the focus of modern linguistics in the Chomskyan tradition has been primarily on the internal dimension (I-language, Chomsky 1986). It has often been seen as part of cognitive psychology, ultimately as a form of theoretical biology. From Lenneberg (1967) to Jenkins (2000), the 'biolinguistic' perspective has been the leading thought. According to this view, a substantial part of language, particularly recursive core grammar, is the abstract description of a 'language organ'. In this view, the human mind and the brain must be identical at some level, witness frequent use of terms like the mind/brain. It is expected (or hoped for), according to the biolinguistic perspective, that ultimately the study of I-language can, at least in principle, be unified with the core natural sciences (biology, chemistry, physics). I will refer to this standard view as linguistic naturalism.

Linguistic naturalism is not without critics, particularly outside the community of generative grammarians in the strict Chomskyan tradition. Thus, many continue to adhere to the traditional, cultural view of language, according to which language primarily is part of our cultural record and external to the human mind (E-language). Another important E-perspective on language is the linguistic Platonism advocated by the late Jerrold Katz and others (see, for instance, Katz 1990 and the works cited there).

Personally, I think a fully naturalistic approach even to core grammar is untenable. I am a moderate Platonist and also committed to a version of the traditional view that language is in the first place a cultural phenomenon, crucially depending on a supra-individual, external record. I furthermore believe that the mind, unlike the brain, cannot be seen in isolation from the shared, external memory with which the brain lives in symbiosis (see Donald 1991). This external, cultural record determines how our biologically given resources are applied. A good example is playing the piano. It is of course based on our biological capacities: tone perception, 
memory, the physiological and neurological aspects of finger movement, etc. Nevertheless, piano playing is primarily seen as a cultural activity. It involves a tradition of musical composition and, crucially, the invention of an artefact, the piano, which allows us to integrate our biological capacities with the culture we participate in.

Clearly, without the invented artefact and tradition, there would not be anything biological that could be reasonably called 'the biology of piano playing.' There have been numerous cultures without pianos, all with people similarly endowed as we from a biological point of view. In general, biology is not culturally transparent. Cultural phenomena heavily depend on our biological capacities, but cannot be 'read back' into our biology. Doing so, was the big mistake of sociobiology and also of its successor, evolutionary psychology, which claims that our biology is transparent with respect to the cultural exigencies of hunter-gatherer societies.

As I have argued elsewhere (Koster 1989), a fully biolinguistic perspective suffers from the same confusion. We can only speak of language because of the invention of cultural artefacts, words, which are comparable to the piano. Only thanks to the interfacing properties of our cultural invention we can speak of language. Words connect our abstract computational facilities with our concepts, neither of which have anything to do with language in abstraction from our invented interface elements. As for the relation between biology and culture, I see no logical difference between playing the piano (and the rest of our culture) and the use of language. Language is of course easier to acquire (no doubt facilitated by evolution) and based on much more accessible structures than playing the piano, but that is irrelevant for the logic of the situation. All cultural phenomena differ in this respect among each other. Thus, singing a simple song involves much more accessible and universally available biological capacities than playing, say, Bach's Goldberg Variations.

In what follows, I will focus on another possibly external and important aspect of language, the Platonic world of universals. Like the argument from culture, the reality of universals leads to the conclusion that linguistics cannot be reduced to, or unified with, the natural sciences. Natural language, then, is E-language in two crucial aspects: next to our mind-external cultural record, it accesses abstract external structures, just like mathematics. 


\section{Universals and particulars}

We live in a dual world: on the one hand we have the particulars of the physical world and on the other hand we have the world of general terms, which determine our thought processes. Even small children understand the distinction 'type-token' as they apply terms like 'dog' to each dog, not just to one dog at a particular place or time. Even proper names, like 'Mary', are universals, since we apply the name in a potentially infinite number of circumstances in which Mary appears somehow. The world of our thought is exclusively based on universals, while the physical world consists of particulars.

This distinction between universals and particulars has always been one of the core problems of philosophy: how are particulars related to universals? The classical answers were given by Plato and Aristotle. According to Plato, universals (or forms) have an existence independent of particulars and the human mind (universalia ante res). Particulars, in this view, are intelligible to the extent that they can be seen as imperfect instantiations of universals accessed by the mind. The universal forms are timeless and necessary and, therefore, even constrain the creator (or whatever determines 'becoming' in our world). Platonism entails a dualistic, two-world ontology.

Plato's student Aristotle thought that Plato's theory was not parsimonious enough and that there was only one world. He furthermore criticized Plato for failing, given his two world-view, to elucidate how the two worlds (of universals and particulars) were connected. This second criticism is certainly to the point, but Aristotle's alternative theory was a dismal failure, causing philosophical confusion until the present day. Basically, Aristotle denied the dual nature of our reality, making him, at least in Western philosophy, the father of one-world ontologies. He sought to overcome the perceived problems with Platonism by reinterpreting universals as somehow being part of particulars (universalia in rebus). This, however, does not make sense.

Since we are talking about a philosophical debate of over 2000 years, there is no point in even beginning to summarize the objections raised against Aristotelianism over time. So, I will limit myself to a very few personal favorites, often without remembering exactly where I learned about them for the first time.

First of all, by making universals part of each particular, the number of universals becomes equal to the number of particulars. This seems to annihilate the concept of a universal somehow. Furthermore, even in the 
simplest cases universals involve properties that are not instantiated by particulars in any obvious sense. Consider a simple geometric form, like a straight line. The length of a straight line can be infinite and therefore be longer than any line found in the physical universe. Similarly, a geometrical straight line has no specific thickness, while each physically realized line has. In fact, the vast majority of mathematical structures has no realization in the world of physical particulars. The same is true for fictional universals like 'unicorns'.

One could of course extend the world of particulars from physical particulars to non-physical particulars and claim that universals define a potential world distinct from the actual, physical world. But in that case the argument against Plato's two-world vision would collapse: Aristotle would have to assume more than one world as well.

Universals are not part of the physical world but perfect forms that are normative with respect to the often less than perfect physical world. Crystals, for instance, show geometrical shapes and are said to be more or less perfect, depending on the extent to which they approach the ideal. More generally, we can say that universals, in relation to physical objects, are not belonging to the object world itself, but to the meta-world in which things are compared with each other and with some standard external to the objects at issue. Aristotelianism, it seems, is a hopeless conflation of objectlevel perspectives and meta-level perspectives.

The intense medieval debate on universals left Western philosophy since the $17^{\text {th }}$ century with a heritage of two major world views: rationalism and empiricism. European rationalism, much inspired by Descartes and culminating in the philosophy of Kant, can be seen as an epistemologization of Platonism. Classical Platonism is ontological, i.e., it postulates the reality of universals in a realm independent of the human mind. According to European rationalism, there is no certainty beyond the human mind and its categories of understanding. Arguably, also Frege's alleged Platonism is of this epistemological kind, which was influenced by Kant but particularly by the $19^{\text {th }}$-century German philosopher Hermann Lotze (see Sluga 1980).

However, it should be stressed that European rationalism is not naturalistic, in the sense that it explicitly denies that our categories of understanding can be reduced to empirical psychology or biology. Famous in this respect is Husserl's attack on psychologism in mathematics, which was inspired by Frege's critique on Husserl's earlier work (see Baker and Hacker 1984: pp. $41 \mathrm{ff}$.). It is very interesting from this perspective that Chomsky, on the one hand, sees his work in the tradition of European 
rationalism, while, on the other hand, he advocates a naturalistic, psychological/biological approach to language and perhaps to cognition in general.

Before going into this matter, I would like to say that, although I accept the critique on psychologism, I do not find said epistemologization of Platonism convincing in the long run. Motivated by a now outdated quest for certainty, it seems to pull the whole world too much within the purview of the human mind. By way of reductio ad absurdum, this easily leads to the megalomanic subjectivism of solipsistic Idealism, at the cost of the common sense view that there exists an objective world independent of the human mind. Whatever consequences were drawn from Descartes' cogito ergo sum, they do not seem to be less hypothetical and remote from absolute certainty than the working hypothesis that a fullfledged world existed before (and will exist after) the short period of time I am aware of it. If we want to be Platonists, I do not see strong reasons to deviate from the original, ontological version.

So, where does the current idea come from that human cognition (including language and, perhaps, mathematics) must be studied from a fully naturalistic point of view? In my opinion, it is a residue of empiricism and materialism, which were (and are) ideologically motivated world views, which are often -wrongly- seen as the core of the scientific world picture.

For empiricists (and their nominalistic predecessors) the only reality is the reality of particulars, which for them creates 'the problem of universals.' This is a curious metaphysical turn, because in a sense, if anything, there is a problem of particulars. Universals are more elementary in our experience, because they are the immediate building blocks of our thoughts and our theories. Particulars are not immediately given in experience but, in the physical sciences, form the hypothetical reality that our thoughts and theories are about.

So, what is the status of universals in a metaphysical framework that only recognizes particulars as real? Traditionally, empiricists have seen universals either as convenient fictions or, at best, as somehow derived from particulars. I will argue in a minute that full naturalism in cognitive science is based on a variant of the empiricist idea that universals can be derived from particulars. The standard approaches to the problem sought to explain the derivation of universals by mysterious processes with names like 'abstraction', 'generalization' or 'induction'. To say that general terms (universals) are derived by 'generalization' is entirely circular, in the spirit of the doctor in Molière's Le malade imaginaire, who claims that opium 
causes sleep thanks to the fact that it contains a substance called vis dormativa 'sleep force'. Since there are no successful ways to derive universals from particulars, empiricists tend to be in denial about universals, calling them 'fictions', etc.

I will take the reality of universals for granted here, as do Chomsky and many other current advocates of naturalism in cognitive science. Granting that full reductionism is unlikely, latter day naturalists believe that, at least in principle, it is possible to unify theories about physical reality with theories about our mental capacities. Note, however, that unification in the physical sciences, say between physics and chemistry, involves theories that are all exclusively about particulars. This is an area where empiricists and rationalists agree, because for empiricists particulars are the only reality to begin with, whereas rationalists see physical phenomena also as particulars, be it as instantiations of universals.

Full unification of physical and cognitive science would involve something totally unheard of, namely unification of theories about particulars (physics) and theories about universals (for instance, linguistics). It would entail the belief that causal chains of particulars could along the way somehow mysteriously produce the universals of our mind. But this 'causal chain' view would be just a variant of empiricist notions like generalization, abstraction or induction and therefore, in my opinion, doomed to be a failure. In other words, the causal chain (what naturalism comes down to) is the latest guise of the Aristotelian error to see particulars and universals in one plane of reality.

Actually, as argued above, thinking and the world of universals are fundamentally different from the physical world of particulars in that thinking involves a meta-perspective on the physical world. Unification of an object perspective (physical sciences) and our meta-perspective seems impossible and we therefore seem to be destined to remain dualists-inpractice for ever (see Bracken 2002 for a related view). This dualism-inpractice should not be confused with classical substance dualism. If there is an epistemological barrier to see the physical world and the meta-world of thought in one plane, the ontological question of substances does not even arise.

Given our epistemological limitations to understand the relation between universals and particulars, belief in full naturalism in cognition is a form of dogmatic monism and in fact an unprovable metaphysical position, an unsubstantiated assumption about the ultimate nature of reality. This is as pointless, I believe, as all other beliefs about the total of reality, like materialism, idealism, or the idea that all is text (certain postmodernists) or 
created by God (most religion). If one dogmatically insists on full naturalism, it can only seemingly be upheld by making a distinction between 'problems and mysteries', the mysteries being a term for what is behind our epistemological horizon (Chomsky 1975: ch.4).

It seems to me that the relation between universals and particulars is a prototypical example of such a rational mystery. Aristotelianism and its nominalistic and empiricist offspring failed to make universals intelligible 'from below', i.e., by starting from particulars. Platonism sees the relation 'from above', i.e., by assuming the distinct reality of universals and by seeing particulars as imperfect instantiations of these universals. I agree about the distinct reality of universals. But is Platonism successful as a theory about the relation between universals and particulars? Ultimately, the relation is a mystery for Plato as well, because he only gives metaphorical and mythological hints, like the myth of the copies seen in the cave or the idea that our mental access to universals is a matter of reminiscence of an earlier life.

Rationalists criticize empiricists for their failure to account for universals on the basis of particulars. But I think it is often overlooked that the problem situation is symmetrical: there is no successful account of the relation seen 'from above' either. For me at least, Platonism is the most successful perspective on universals, but it leaves it a complete mystery how our brain can access these universals and how they can be recognized in particulars.

As soon, then, as one (against the empiricists) believes in the reality of universals, the distinction between 'naturalism cum mysteries' (Chomsky) and 'Platonism cum mysteries' (Katz) should not be exaggerated. I see Chomsky's naturalism as a kind of naturalized Kantianism, in which the Kantian objections against a naturalistic approach of our categories of thought are met by the introduction of an epistemological horizon (the 'mysteries' of Chomsky 1975: ch. 4). But Kantianism, it should be remembered, was the culmination of the century-long attempts of European rationalism to replace Plato's ontology by a more epistemological perspective. As argued above, this epistemological turn was inspired by an ill-conceived quest for philosophical certainty, which, I believe is ultimately futile.

\section{Concluding remarks}

Given our limited epistemological horizon, in practice, the main difference between Platonism and naturalism will be that according to the Platonist, 
the brain somehow accesses the (external) universals, while the naturalist will say that universals are mind-internal and caused by the brain. I doubt if these two positions are empirically distinguishable and, if I am correct, they both run into the barrier that prevents us from seeing universals and particulars in a unified, one-world theory. This leaves us minimally, once more, as dualists-in practice, with no definitive conclusion about the ultimate nature of our reality.

If we look at our scientific practice, the dual nature of our reality is confirmed by the existence of mathematics. Not the physical sciences, but mathematics is our most successful form or rational inquiry. The development of mathematics looks like the development of the empirical sciences in important respects, but is about universals and not about particulars. In this sense, it is a Platonic science and mathematics has, understandably, always been the field of choice of the Platonically inclined. We need mathematics to understand the physical world, not the other way around. Naturalism, in the sense of reduction or unification of mathematics with physics, is not even considered.

Why would naturalism be more plausible with respect to linguistic computation than with respect to the patterns of necessity studied by mathematics? Like mathematics, linguistics studies universals, such as our concepts or the recursive structures of grammar ('merge'). Recursion is an instance of self-similarity, a form of symmetry ubiquitous in the extramental, physical world. Patterns of symmetry are the subject matter par excellence of mathematics. Hence, I believe that, as for concepts and computations, linguistics is a form of inquiry in the same league as mathematics, studying universals rather than the particulars that are the subject matter of the natural sciences.

This view also seems to be confirmed by the actually existing brain sciences. At best so far, we are successful with localizations, i.e., by determining the part of the brain where certain computations take place. But as we know from computers, indicating where in some hardware device computation takes place is very remote from unifying the theory of the computation and the physics of the hardware. Similarly, it is an error and an illusion to believe that the brain sciences -naturalistic theories about particulars- can explain our thought processes based on the universals studied by mathematic and linguistics. As in the relation between mathematics and physics, I believe that linguistics is like mathematics in that it is more fundamental than whatever the brain sciences come up with. Linguistics can guide us in understanding where and how the brain executes certain computations, but what makes computations computations 
(or, for that matter, what makes a triangle or a number a triangle or a number) is beyond the possible scope of the natural sciences.

\section{References}

Baker, Gordon, and Peter Hacker. 1984. Frege: Logical Excavations. Oxford, etc.: Oxford University Press.

Bracken, Harry. 2002. Descartes. Oxford: One World.

Chomsky, Noam. 1975. Reflections on Language. New York: Pantheon Books.

Chomsky, Noam. 1986. Knowledge of Language. New York: Praeger.

Donald, Merlin. 1991. Origins of The Modern Mind. Cambridge, Mass.: Harvard University Press.

Jenkins, Lyle. 2000. Biolinguistics: Exploring the Biology of Language. Cambridge: Cambridge University Press.

Katz, Jerrold . 1990. The Metaphysics of Meaning. Cambridge, Mass.: MIT Press.

Koster, Jan. 1989. How Natural is Natural Language? in J.E. Fenstad et al. (eds.), Logic, Methodology, and Philosophy of Science VIII. Amsterdam, etc.: Elsevier.

Lenneberg, Eric. 1967. Biological Foundations of Language. New York: John Wiley and Sons.

Sluga, Hans. 1980. Gottlob Frege. London, etc.: Routledge and Kegan Paul. 